phys. stat. sol. (a) 166, 429 (1998)

Subject classification: 71.15.Mb; 61.85.+p ; 62.20.Dc ; 71.20.Ps; S10

\title{
Electronic Structure and Elastic Properties of Strongly Correlated Metal Oxides from First Principles: LSDA + U, SIC-LSDA and EELS Study of $\mathrm{UO}_{2}$ and $\mathrm{NiO}$
}

\author{
S. L. Dudarev (a), G. A. Botton (b), S. Y. Savrasov (c), Z. Szotek (d), \\ W. M. Temmerman (d) and A. P. Sutton (a)
}

(a) Department of Materials, University of Oxford, Parks Road, Oxford OX1 3PH, UK

(b) Department of Materials Science and Metallurgy, University of Cambridge, Pembroke Street, Cambridge CB2 3QZ, UK

(c) Max-Planck-Institut für Festkörperforschung, Heisenbergstrasse 1, D-70569 Stuttgart, Germany

(d) Daresburg Laboratory, Warrington WA4 4AD, UK

(Received November 5, 1997)

Dedicated to Professor Dr. ManfRed RüHLE on the occassion of his 60th birthday

We compare experimentally observed electron energy loss spectra (EELS) of uranium dioxide $\mathrm{UO}_{2}$ and nickel monoxide $\mathrm{NiO}$ with the results of ab-initio calculations carried out by using a method combining the local spin density approximation and the Hubbard U term (the LSDA + U method). We show that by taking better account of strong Coulomb correlations between electrons in the $5 \mathrm{f}$ shell of uranium ions in $\mathrm{UO}_{2}$ and in the $3 \mathrm{~d}$ shell of nickel ions in $\mathrm{NiO}$ it is possible to arrive at a better description of electron energy loss spectra, cohesive energies and elastic constants of both oxides compared with local spin density functional theory. For NiO we also compare the LSDA + U results and EELS spectra with a self-interaction corrected LSDA calculation.

\section{Introduction}

The problem of first-principles description of electronic properties of transition metal and actinide oxides and their surfaces has recently attracted considerable attention and presently remains one of the most actively studied subjects in solid state physics and materials science. Interest in this problem stems both from numerous applications of transition metal and actinide oxides, notably in heterogeneous catalysis [1, 2], and also from a large variety of unusual electronic effects originating from strong Coulomb repulsion between electrons occupying partly filled $\mathrm{d}$ and $\mathrm{f}$ shells of metal ions in these oxides [3 to 5].

Transition metal and actinide oxides are also known as compounds where traditional ab-initio methods based on the local spin density approximation (LSDA) to the density functional theory (DFT) often fail to predict the experimentally observed insulating ground states. Yamashita and Asano [6] and Terakura et al. [7] have found that for transition metal oxides a DFT-LSDA calculation often leads to a metallic ground state. 
Insulating ground state may be obtained provided that the appropriate antiferromagnetic order associated with polarization of partly filled $d$ shells is taken into accout [8] but the forbidden gap in DFT-LSDA still turns out to be much smaller than that observed using electron spectroscopy [9].

Uranium dioxide represents a similar example of a compound where uranium ions contain partly filled f shells and where the DFT-LSDA ground state is metallic $[10,11]$. Besides that, DFT-LSDA systematically underestimates equilibrium lattice constants of both transition metal and actinide oxides and overestimates their binding energies $[12,10,11]$, raising questions about the applicability of the DFT-LSDA approach to making predictions concerning equilibrium configurations of surface and defect structures.

Until recently the electronic structure of transition metal and actinide oxides was studied experimentally mainly by using X-ray photoelectron spectroscopy (XPS) and inverse photoemission spectroscopy (IPS) $[13,14]$. These two techniques are primarily sensitive to the electronic structure of bulk layers of atoms. Recent progress in experimental elevated temperature scanning tunneling microscopy (STM) has made it possible to perform direct observation of atoms as well as individual defects on insulating metal oxide surfaces [15 to 17]. To interpret STM images of surface atomic layers one needs to develop a computational approach capable of calculating and comparing total energies of various equilibrium configurations of atoms at surfaces as well as evaluating certain spectroscopical quantities, for example, the spectrum of one-electron bulk and surface excitations of the material.

Since an ion situated on a surface has many more degrees of freedom than an ion constituting a part of a three-dimensionally periodic crystal lattice, the problem of $a b$ initio calculation of the electronic structure of a surface turns out to be far more demanding computationally than the corresponding bulk problem. At the same time to understand surface electronic structure of a particular oxide one needs first to clarify all the points relevant to the formation of electronic states in the crystal bulk. In this paper we address the latter problem and show how the effective density of bulk one-electron states observed by electron energy loss spectroscopy (EELS) can be simulated in parallel with a number of parameters characterizing the structural stability of an oxide.

In our analysis we consider several quantities by looking at which one can obtain a reasonably good understanding of how electron correlation effects influence the observed properties of a particular oxide. Our study is based on a recently proposed method combining the local density approximation and the term describing on-site Coulomb repulsion between localized d or f electrons (the LSDA + U approach [18 to 20]). The results described below were obtained by using a full-potential LMTO [21] implementation of the LSDA + U approach. In the case of $\mathrm{NiO}$, we also present the results obtained by using the self-interaction corrected (SIC) LSDA approach to the description of on-site Coulomb correlations between localized d electrons, implemented within the LMTOASA band structure method.

The paper is organized as follows. Firstly, we describe how the LSDA + U method can be formulated in a rotationally-invariant way that displays the correct asymptotic behavior in the limit of large separation between ions and therefore allows one to compare quantities calculated by varying the interatomic distance. In the same section we briefly describe the SiC-LSDA formalism. Then we discuss results obtained both for uranium dioxide and nickel monoxide, and compare the simulated energy loss spectra 
with those observed in a transmission electron microscope. Finally, we show how by varying the effective Hubbard $U$ characterizing the on-site Coulomb repulsion between $5 \mathrm{f}$ electrons in $\mathrm{UO}_{2}$ and between $3 \mathrm{~d}$ electrons in $\mathrm{NiO}$ it is possible to achieve good correspondence between the calculated and experimentally observed values of the lattice constant, the cohesive energy and moduli characterizing elastic properties of the two oxides. For $\mathrm{NiO}$ we also compare the LSDA $+\mathrm{U}$ densities of states and the experimentally observed energy loss spectra with those calculated using the selfinteraction corrected LSDA. Moreover, we present a comparison of the bandgap, magnetic moment, lattice constant and bulk modulus calculated by the two theoretical approaches. We demonstrates that a detailed simulation of energy loss spectra can provide useful information about bonding in transition metal and actinide oxides and that the structure of EELS spectra can be related to the ground state properties of the material.

\section{Calculating Total Energies within LSDA + U and SIC-LSDA}

\section{$2.1 L S D A+U$}

The LSDA + U method proposed by Anisimov et al. [18, 19] and reformulated in a rotationally-invariant form by Liechtenstein et al. [20] represents a convenient way of combining the LMTO implementation of the DFT-LSDA formalism (for a recent review see the paper by Savrasov [21] with the unrestricted Hartree-Fock (UHF) treatment of the Hubbard model. The UHF approximation corresponds to the following simplification of the term describing on-site Coulomb repulsion between electrons [22] $U \hat{n}_{\sigma} \hat{n}_{-\sigma} \approx U \hat{n}_{\sigma} n_{-\sigma}$, where $\hat{n}_{\sigma}=\hat{a}_{\sigma}^{\dagger} \hat{a}_{\sigma}$ is the operator of the number of electrons occupying a particular site and $n_{\sigma}$ is its expectation value calculated in a self-consistent way for the ground state of the system.

To describe Coulomb interaction between $5 f$ electrons localized on uranium sites in $\mathrm{UO}_{2}$ or between $3 \mathrm{~d}$ electrons of nickel ions in $\mathrm{NiO}$ we use the following model Hamiltonian [23]:

$$
\hat{H}=\frac{\bar{U}}{2} \sum_{m, m^{\prime}, \sigma} \hat{n}_{m, \sigma} \hat{n}_{m^{\prime},-\sigma}+\frac{(\bar{U}-\bar{J})}{2} \sum_{m \neq m^{\prime}, \sigma} \hat{n}_{m, \sigma} \hat{n}_{m^{\prime}, \sigma},
$$

where summation is performed over projections of the orbital momentum $\left(m, m^{\prime}=-3\right.$, $-2 \ldots, 3$ in the case of $\mathrm{f}$ electrons) and $\bar{U}$ and $\bar{J}$ are the spherically averaged matrix elements of the screened Coulomb electron-electron interaction.

In the UHF approximation the expectation value of $\hat{H}$ is given by

$$
\left\langle N_{\sigma}|\hat{H}| N_{\sigma}\right\rangle_{\mathrm{UHF}}=\frac{\bar{U}}{2} \sum_{\sigma, m, m^{\prime}} n_{m, \sigma} n_{m^{\prime},-\sigma}+\frac{(\bar{U}-\bar{J})}{2} \sum_{\sigma, m \neq m^{\prime}} n_{m, \sigma} n_{m^{\prime}, \sigma},
$$

where $n_{m, \sigma}$ is the occupation number of a particular $\mathrm{f}$ or $\mathrm{d}$ state. For an integer total number $N_{\sigma}$ of electrons in the shell with a given projection of spin $\sigma$ this expectation value equals

$$
\left\langle\text { integer } N_{\sigma}|\hat{H}| \text { integer } N_{\sigma}\right\rangle=\frac{\bar{U}}{2} \sum_{\sigma} N_{\sigma} N_{-\sigma}+\frac{(\bar{U}-\bar{J})}{2} \sum_{\sigma} N_{\sigma}\left(N_{\sigma}-1\right) \text {. }
$$


The LSDA + U approach is based on the conjecture that (3) represents the correct form of the density functional corresponding to Hamiltonian (1) and that equation (3) retains its validity also for the case of non-integer $N_{\sigma}$. By subtracting (2) from (3) and by adding the result to the conventional LSDA energy functional we arrive at an approach where all the orbitals except those included in the Hamiltonian (1) are treated within the framework of LSDA while the localized $\mathrm{f}$ or $\mathrm{d}$ states are treated by using the UHF approximation. (Note that since below we will be studying a self-consistent solution of LSDA + U equations, strong correlation effects associated with $\mathrm{f}$ or $\mathrm{d}$ states are going to affect all other states as well. In particular this point is relevant to the treatment of oxygen $2 p$ states which, although being not directly influenced by Hubbard correlations, are linked to those states via hybridization terms). In an explicit form the LSDA + U energy functional can be written as $[18,19]$

$$
E_{\mathrm{LSDA}+\mathrm{U}}=E_{\mathrm{LSDA}}+\frac{(\bar{U}-\bar{J})}{2} \sum_{m, \sigma}\left[n_{m, \sigma}-n_{m, \sigma}^{2}\right] .
$$

This equation can be further transformed into a rotationally-invariant form. To do this we notice that sums $\sum_{m} n_{m, \sigma}$ and $\sum_{m} n_{m, \sigma}^{2}$ can be represented as traces of, respectively, $\varrho_{\sigma}$ and $\varrho_{\sigma}^{2}$, where $\varrho_{\sigma} \equiv \varrho_{j l}^{\sigma}$ is the density matrix of electrons occupying f or d shell and $j$ and $l$ are indices distinguishing between different $\mathrm{f}$ or $\mathrm{d}$ orbitals. This makes it possible to turn equation (4) into a rotationally-invariant construction,

$$
E_{\mathrm{LSDA}+\mathrm{U}}=E_{\mathrm{LSDA}}+\frac{(\bar{U}-\bar{J})}{2} \sum_{\sigma}\left[\operatorname{Tr} \varrho^{\sigma}-\operatorname{Tr}\left(\varrho^{\sigma} \varrho^{\sigma}\right)\right]
$$

Note that the second term in the right-hand side of equations (4) and (5) vanishes in the limit of integer partial occupancies $n_{m, \sigma}=0$ or $n_{m, \sigma}=1$ corresponding to large separation between ions. This observation shows that (i) it is not necessary to take the Hubbard correction into account when calculating the total energy of individual atoms constituting the solid (this energy is required as a reference point when calculating the cohesive energy of a solid) and (ii) the Hubbard correction to the total energy associated with the formation of covalent bonds is a positive quantity and therefore the cohesive energy calculated using the above LSDA $+\mathrm{U}$ formalism is going to be lower than the corresponding quantity calculated by means of conventional LSDA.

To complete the discussion of the practical implementation of the LSDA $+\mathrm{U}$, we note that the matrix of the one-electron potential is given by the derivative of (5) with respect to $\varrho_{l j}^{\sigma}$,

$$
V_{j l}^{\sigma} \equiv \frac{\delta E_{\mathrm{LSDA}+\mathrm{U}}}{\delta \varrho_{l j}^{\sigma}}=\frac{\delta E_{\mathrm{LSDA}}}{\delta \varrho_{l j}^{\sigma}}+(\bar{U}-\bar{J})\left[\frac{1}{2} \delta_{j l}-\varrho_{j l}^{\sigma}\right],
$$

and that the total energy can be expressed in terms of the Kohn-Sham eigenvalues $\left\{\varepsilon_{i}\right\}$ as

$$
E_{\mathrm{LSDA}+\mathrm{U}}=E_{\mathrm{LSDA}}\left[\left\{\varepsilon_{i}\right\}\right]+\frac{(\bar{U}-\bar{J})}{2} \sum_{l, j, \sigma} \varrho_{l j}^{\sigma} \varrho_{j l}^{\sigma},
$$

where the last term represents the double counting correction. Below we apply (6) and (7) to calculate the spectrum of one-particle density of states and elastic moduli of uranium dioxide and nickel oxide. 


\section{2 $S I C-L S D A$}

Another approach, known to describe adequately strong electron correlations in transition metal oxides, is the SIC-LSDA [24]. In the SIC-LSDA one starts from the LSD total energy functional, but corrects for the self-Coulomb and self-exchange-correlation energy of each of the occupied electron states, which erroneously is included in the LSD approximation. Specifically,

$$
\begin{aligned}
\tilde{E}^{\mathrm{SIC}}\left[\left\{\psi_{\alpha}\right\}\right]= & \sum_{\alpha}\left\langle\psi_{\alpha}|-\Delta| \psi_{\alpha}\right\rangle+U[n]+E_{\mathrm{xc}}^{\mathrm{LSD}}[\bar{n}]+V_{\mathrm{ext}}[n] \\
& -\sum_{\alpha}\left\{U\left[n_{\alpha}\right]+E_{\mathrm{xc}}^{\mathrm{LSD}}\left[\bar{n}_{\alpha}\right]\right\} .
\end{aligned}
$$

Here, $\tilde{E}^{\text {SIC }}$ is a functional of a set of $N$ occupied orthonormal single electron wavefunctions $\psi_{\alpha} \cdot \bar{n}$ is the total spin density of the system, $\bar{n}(\mathbf{r})=\left(n^{\uparrow}(\mathbf{r}), n^{\downarrow}(\mathbf{r})\right), n(\mathbf{r})=n^{\uparrow}(\mathbf{r})$ $+n^{\downarrow}(\mathbf{r})$ and $\bar{n}_{\alpha}$ is the spin density of the $\alpha$ th state, $\bar{n}_{\alpha}(\mathbf{r})=\left(\left|\psi_{\alpha}(\mathbf{r})\right|^{2}, 0\right),\left(0,\left|\psi_{\alpha}(\mathbf{r})\right|^{2}\right)$, for spin up and spin down states, respectively. $U[n]$ and $E_{x c}^{\mathrm{LSD}}[\bar{n}]$ are the Coulomb and the exchange-correlation energies of the electron gas, respectively, while $V_{\text {ext }}[n]$ denotes the interaction energy with the lattice of ions,

$$
\begin{aligned}
& U[n]=\iint \frac{n(\mathbf{r}) n\left(\mathbf{r}^{\prime}\right)}{\left|\mathbf{r}-\mathbf{r}^{\prime}\right|} \mathrm{d}^{3} r \mathrm{~d}^{3} r^{\prime}, \\
& E_{\mathrm{xc}}^{\mathrm{LSD}}[\bar{n}]=\int \varepsilon_{\mathrm{xc}}^{\mathrm{hom}}(\bar{n}(\mathbf{r})) n(\mathbf{r}) \mathrm{d}^{3} r, \\
& V_{\text {ext }}[n]=\int V_{\text {ext }}(\mathbf{r}) n(\mathbf{r}) \mathrm{d}^{3} r .
\end{aligned}
$$

In Eq. $(10), \varepsilon_{\mathrm{xc}}^{\text {hom }}(\bar{n})$ is the exchange-correlation energy density of a homogeneous electron gas at spin density $\bar{n}[25]$, for which we adopt the parametrization as given by Perdew and Zunger [24].

The last term in Eq. (8) represents the self-interaction correction, where for each occupied orbital $\psi_{\alpha}$ the Coulomb and exchange-correlation energies of the corresponding spin density $\bar{n}_{\alpha}$ is subtracted. If the orbitals in the kinetic energy term are taken to be Kohn-Sham orbitals and the self-interactions is omitted we would have the LSDA energy functional $E^{\mathrm{LSD}}$.

The SIC-LSDA restores the property, which the true density functional has, that for any single-electron density $\bar{n}_{\alpha}$,

$$
U\left[n_{\alpha}\right]+E_{\mathrm{xc}}\left[\bar{n}_{\alpha}\right]=0 .
$$

This does not hold for the LSDA.

The SIC-LSDA has been widely applied to atoms, molecules, clusters, impurities and solids (see Temmerman et al. [26] for a comprehensive list of references). In particular, for periodic solids the SIC-LSDA constitutes an extension of LSDA in the sense that the self-interaction correction is finite only for electron states that are spatially localized. This means that if the single-particle states are restricted to be of Bloch type, $\tilde{E}^{\text {SIC }}$ coincides with $E^{\mathrm{LSD}}$, i.e., the LSDA minimum is also a local minimum of $\tilde{E}^{\mathrm{SIC}}$. The question is, whether a set of single-particle states, not all of Bloch form, exists such that $\tilde{E}^{\text {SIC }}$ has a lower energy when evaluated with these orbitals. This line of thought was pursued in an investigation of a body-centered cubic array of hydrogen atoms [27], 
where a discontinuous Mott transition was predicted as a function of lattice spacing, precisely as a consequence of the Bloch-like (LSD) local minimum and the localized (SIC-LSDA) minimum shifting relatively to each other. Consequently, the concept of localization in the SIC-LSDA scheme becomes very literal, the 'localized' states being really spatially confined. Since this study, localization phenomena have been investigated by the SIC-LSDA approach in the $3 \mathrm{~d}$ transition metal oxides [28 to 32 ] and rare earths (see Temmerman et al. [26] and references therein).

All the results for $\mathrm{NiO}$ described below have been obtained using the LMTO-ASA implementation of the SIC-LSDA approach. This was done in the screened representation and with the combined correction term included [33]. A minimal basis set of Ni s,p,d low waves and intermediate $\mathrm{f}$ wave together with $\mathrm{O}$ s,p low waves and intermediate $\mathrm{d}$ wave was used.

\section{Comparison of Calculated and Observed Properties of $\mathrm{UO}_{2}$}

We start by comparing the experimentally observed electron energy loss spectrum of $\mathrm{UO}_{2}$ with the results of LSDA $+\mathrm{U}$ calculations. The specimens for the EELS experiments were prepared by crushing the $\mathrm{UO}_{2}$ crystals in pure ethanol and subsequent drying the dispersion onto a lacey carbon film deposited on a TEM Cu grid. The EELS measurements were carried out on a dedicated scanning transmission electron microscope equipped with a Gatan Imaging Filter (UHV GIF model 678). The system uses a cold field emission gun and permits an energy resolution of about 0.4 to $0.5 \mathrm{eV}$ (as measured at the full width at half maximum of the zero loss peak). Spectra obtained from single grains of the powder were background subtracted and corrected for gain variation of the detector as described in [34]. The near edge structure of the $\mathrm{O} \mathrm{K}$ spectrum of $\mathrm{UO}_{2}$ obtained in such a way and shown in Fig. 1 represents the transitions from the occupied
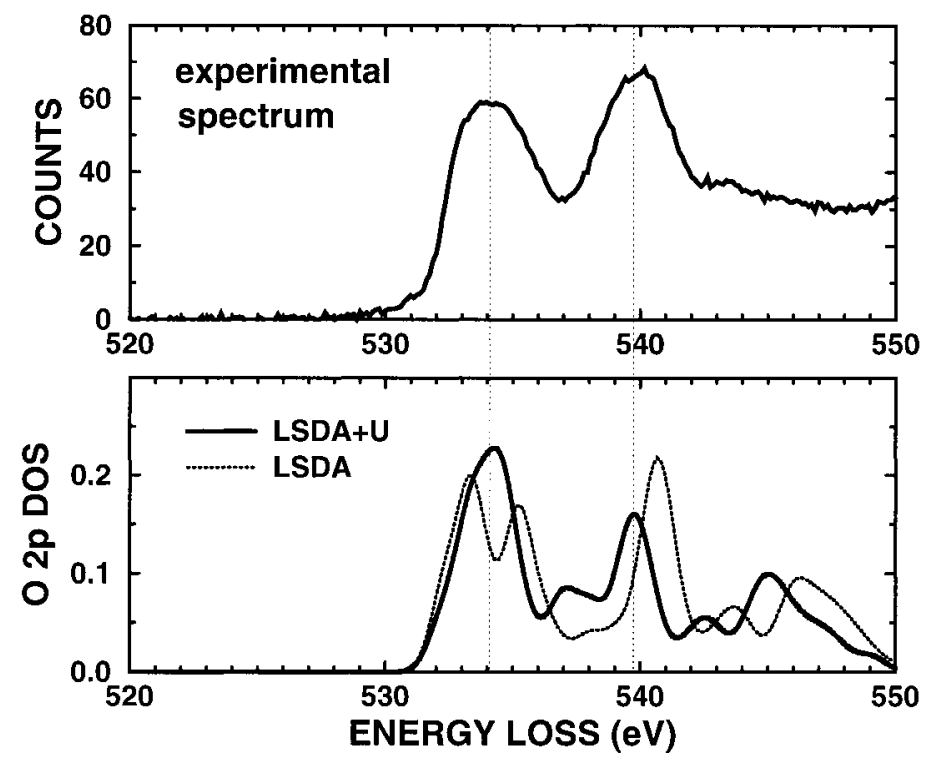

Fig. 1. Experimental electron energy loss oxygen $\mathrm{K}$ edge spectrum of $\mathrm{UO}_{2}$ and the density of empty oxygen $2 \mathrm{p}$ states of $\mathrm{UO}_{2}$ calculated by using LSDA + U and LSDA 
O 1s core levels to the conduction band states. Due to dipole selection rules and the necessary overlap of the initial and final states for the transition to be observed (i.e. non-zero transition matrix elements) the final states probed are of $\mathrm{p}$ symmetry and are localized on the oxygen sites.

Numerical calculations were performed using the method described in the preceding section and implemented on the basis of the full-potential LMTO program described in [21]. The densities of one-particle electron states (DOS) were calculated using three energy panels and $7 \times 7 \times 7=343 k$-points in the entire Brillouin zone. Since the structure of the experimental EELS spectrum shown in Fig. 1 is dominated by the dipole-allowed \{filled $1 \mathrm{~s}\} \rightarrow\{$ empty $2 \mathrm{p}\}$ transitions, to simulate the experimental spectrum the oxygen $2 \mathrm{p}$ DOS were broadened with a Gaussian distribution to account for the lifetime of the excited state and the instrumental response of the system (resolution and energy spread of the primary electrons). This method has already been applied to simulations of $\mathrm{L}$ and $\mathrm{K}$ edges in semiconductors [35] and $\mathrm{O} \mathrm{K}$ edges in early transition metal oxides [36]. The spectrum of oxygen empty 2p DOS calculated using $\bar{U}=4.5 \mathrm{eV}$ and $\bar{J}=0.51 \mathrm{eV}$ is shown in Fig. 1. The above values of $\bar{U}$ and $\bar{J}$ were determined by using experimental XPS data reported by Kotani and Yamazaki [23] and results of optical spectroscopy measurements performed by Krupa and Gajek [37]. To find $\bar{U}$ and $\bar{J}$ we used the following equation relating these quantities to the Slater integrals $F^{0}, F^{2}, F^{4}$ and $F^{6}$ of $5 \mathrm{f}$ shell of uranium ions,

$$
\begin{aligned}
& \bar{U}=F^{0}, \\
& \bar{J}=\frac{1}{3}\left(\frac{2}{15} F^{2}+\frac{1}{11} F^{4}+\frac{50}{429} F^{6}\right),
\end{aligned}
$$

where [37] $F^{2}=5.34 \mathrm{eV}, F^{4}=5.08 \mathrm{eV}$ and $F^{6}=2.95 \mathrm{eV}$. Equation (13) represents a particular case of a more general equation given by Griffith [38] and valid for any electronic shell,

$$
\begin{aligned}
\bar{U}-\bar{J}= & \sum_{k=0,2,4 \ldots}^{2 l} F^{k} \\
& \times\left\{\frac{1}{(2 l+1) 2 l} \sum_{m, m^{\prime}}\left[c^{k}(l m, l m) c^{k}\left(l m^{\prime}, l m^{\prime}\right)-c^{k}\left(l m, l m^{\prime}\right) c^{k}\left(l m, l m^{\prime}\right)\right]\right\},
\end{aligned}
$$

and $c^{k}\left(l m, l m^{\prime}\right)$ are the Gaunt parameters [38]. In agreement with the results of previous LSDA + U calculations [11], the ground state of uranium dioxide in LSDA + U is antiferromagnetic and insulating with the $1.4 \mathrm{eV}$ bandgap separating two bands of uranium $5 \mathrm{f}$ states (the size of the bandgap observed experimentally is slightly larger [39] and is close to $2 \mathrm{eV}$ ). In LSDA uranium dioxide is known to be metallic [10,11], a result that disagrees with experimental observations [39, 17]. In LSDA + U the ground state of uranium dioxide is insulating. Coulomb repulsion between $5 \mathrm{f}$ electrons localized on uranium sites leads to splitting of occupied and unoccupied $5 \mathrm{f}$ bands. Since this splitting does not alter the sequence of bands, uranium dioxide can be classified as a Mott-Hubbard insulator [23, 11].

In Fig. 1 also shown is the spectrum of oxygen empty 2p DOS calculated by using the values of $\bar{U}$ and $\bar{J}$ which were reduced ten times in comparison with those used in the above LSDA + U calculation (we were not able to obtain a stable FP-LMTO solution in 
the limit $\bar{U}=0$ and $\bar{J}=0$ but we believe that the result obtained using very small values of $\bar{U}$ and $\bar{J}$ is sufficiently close to this limit). An examination of Fig. 1 shows that apart from the change in the magnitude of peaks, the basic difference between the LSDA + U and the LSDA solutions is associated with the change in the separation between the two main peaks visible in the two spectra. These peaks result from hybridization between oxygen $2 \mathrm{p}$ and uranium $5 \mathrm{f}$ and uranium $6 \mathrm{~d}$ states, respectively. In the LSDA + U solution the distance between the two peaks equals $5.5 \mathrm{eV}$ while in the LSDA solution these peaks are separated by the interval of $6.5 \mathrm{eV}$ (in the case of LSDA the lower peak is further split into two smaller peaks which are not resolved in the experimental spectrum shown in Fig. 1). The separation between the two main peaks in the experimental spectrum equals $5.8 \mathrm{eV}$ and this value is closer to the LSDA + U result than to the LSDA value.

Further evidence pointing to the important role played by Coulomb repulsion between 5f electrons is provided by the data displaying how the parameters characterizing the electronic and crystal structures of $\mathrm{UO}_{2}$ vary under external pressure. Fig. 2 shows the bandgap separating $5 \mathrm{f}$ states, the magnetic moment associated with uranium sites and the total energy of the antiferromagnetic unit cell (containing two uranium and four

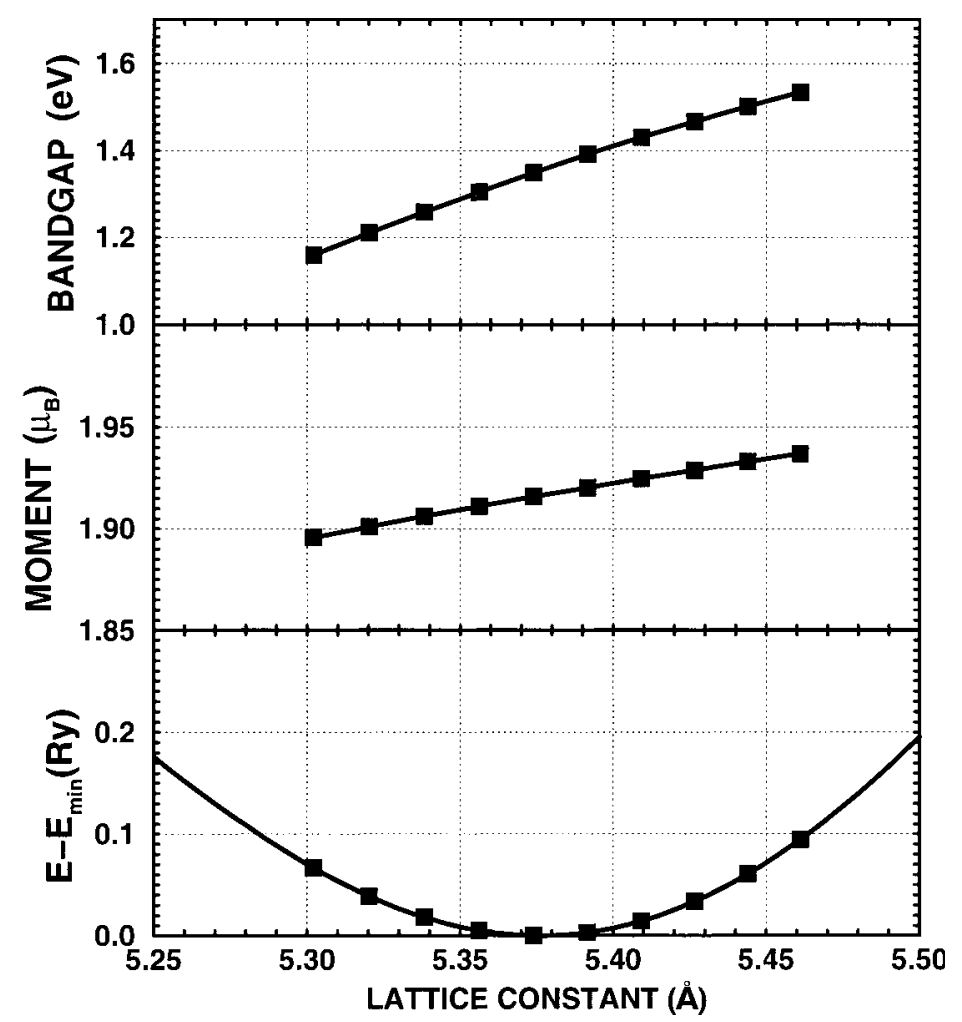

Fig. 2. The bandgap, the spin magnetic moment of a uranium ion in $\mathrm{UO}_{2}$ and the total energy of the antiferromagnetic unit call plotted as a function of the lattice constant. The bandgap of $\mathrm{UO}_{2}$ observed by using X-ray photoelectron spectroscopy [39] equals $2 \mathrm{eV}$, the magnetic moment of a uranium ion in $\mathrm{UO}_{2}$ is [43] $1.74 \mu_{\mathrm{B}}$ and the equilibrium lattice constant equals $5.46 \AA$ 
Table 1

Calculated and observed values of parameters characterizing the electronic structure of crystalline $\mathrm{UO}_{2}$ and $\mathrm{NiO}$ and their structural stability. The Hartree-Fock values and experimental data for $\mathrm{NiO}$ were taken from [47]

\begin{tabular}{|c|c|c|c|c|c|}
\hline quantity versus method & LSDA & $\mathrm{LSDA}+\mathrm{U}$ & SIC-LSDA & $\begin{array}{l}\text { Hartree- } \\
\text { Fock }\end{array}$ & $\begin{array}{l}\text { observed } \\
\text { value }\end{array}$ \\
\hline lattice constant of $\mathrm{UO}_{2}(\AA)$ & 5.19 & $5.37[\bar{U}=4.5 \mathrm{eV}]$ & - & - & 5.46 \\
\hline lattice constant of $\mathrm{NiO}(\AA)$ & 4.08 & $4.19[\bar{U}=6.2 \mathrm{eV}]$ & 4.18 & 4.26 & 4.17 \\
\hline bandgap of $\mathrm{UO}_{2}(\mathrm{eV})$ & absent & 1.3 & - & - & 2.0 \\
\hline bandgap of $\mathrm{NiO}(\mathrm{eV})$ & 0.6 & 3.0 & 3.15 & 14.2 & 4.2 \\
\hline cohesive energy of $\mathrm{UO}_{2}(\mathrm{eV})$ & 27.48 & 22.23 & - & - & 22.31 \\
\hline cohesive energy of $\mathrm{NiO}(\mathrm{eV})$ & 13.74 & 11.60 & - & 6.2 & 8.26 \\
\hline $\begin{array}{l}B=\left(C_{11}+2 C_{12}\right) / 3 \text { of } \mathrm{UO}_{2} \\
(\mathrm{GPa})\end{array}$ & $\left.233^{\mathrm{a}}\right)$ & 173 & - & - & 207 \\
\hline $\begin{array}{l}B=\left(C_{11}+2 C_{12}\right) / 3 \text { of } \mathrm{NiO} \\
\quad(\mathrm{GPa})\end{array}$ & $\begin{array}{l}230 \\
\left.236^{\mathrm{b}}\right)\end{array}$ & 182 & 220 & 214 & $145,205,189$ \\
\hline $\begin{array}{l}C^{\prime}=\left(C_{11}-C_{12}\right) / 2 \text { of } \mathrm{UO}_{2} \\
(\mathrm{GPa})\end{array}$ & - & 117 & - & - & 137 \\
\hline $\begin{array}{c}C^{\prime}=\left(C_{11}-C_{12}\right) / 2 \text { of } \mathrm{NiO} \\
(\mathrm{GPa})\end{array}$ & 211 & 161 & - & 136 & 45,102 \\
\hline$C_{44}$ of $\mathrm{UO}_{2}(\mathrm{GPa})$ & - & 149 & - & - & 64 \\
\hline$C_{44}$ of $\mathrm{NiO}(\mathrm{GPa})$ & 49 & 86 & - & 115 & 110,105 \\
\hline
\end{tabular}

a) Value calculated by using the atomic sphere approximation (ASA) [11].

b) Value calculated by Sasaki [48].

oxygen ions) as a function of the lattice constant. Although both the bandgap and the magnetic moment decrease with increasing pressure, they remain finite at the point where the total energy is a minimum. The calculated equilibrium value of the lattice constant $a_{0}=5.37 \AA$ is close to the experimentally observed value of $a_{0}^{(\exp )}=5.46 \AA$. The cohesive energy of $\mathrm{UO}_{2}$ obtained by comparing the minimum of the curve shown in Fig. $2\left(E_{\min }=-112872.698 \mathrm{Ry}\right)$ with the energies of individual uranium and oxygen ions $E_{\mathrm{U}}=-56136.605 \mathrm{Ry}$ and $E_{\mathrm{O}}=-149.055 \mathrm{Ry}$ equals $E_{\mathrm{coh}}=1.63 \mathrm{Ry}$ and is also very close to the experimental value of $E_{\text {coh }}^{(\exp )}=1.64$ Ry determined by Kelly and Brooks

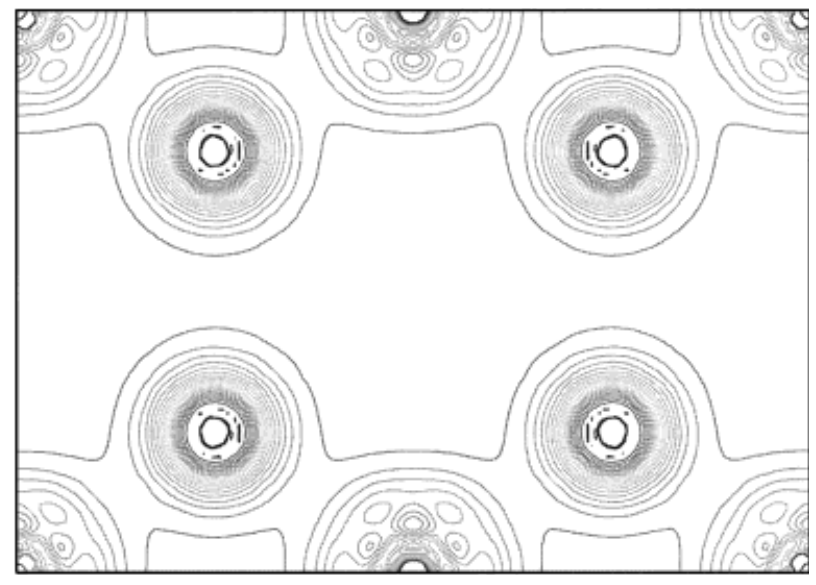

Fig. 3. The distribution of the total charge density in the (110) plane of $\mathrm{UO}_{2}$ calculated by the $\mathrm{LSDA}+\mathrm{U}$ approach 
[40]. Elastic moduli of $\mathrm{UO}_{2}$ were calculated following the procedure described by Sherman [41]. The calculated values are listed in Table 1 together with the corresponding experimental data taken from [42].

To complete the discussions of bulk properties of uranium dioxide and to provide a way of comparing this actinide oxide with a transition metal oxide $(\mathrm{NiO})$ described in the following section, in Fig. 3 we plotted the distribution of charge density in the (110) plane calculated using the LSDA $+\mathrm{U}$ approach. In Fig. 3 six uranium ions occupy positions along the edges of the picture. Four oxygen ions are shown closer to the centre of the image. Note that the distribution of charge around metal and oxygen ions in $\mathrm{UO}_{2}$ is almost spherical. Charge density distribution of the form shown in Fig. 3 represents a directly measurable quantity as has been recently demonstrated by Zuo et al. [44] for $\mathrm{MgO}$. Zuo et al. also found the charge distribution around magnesium and oxygen ions in $\mathrm{MgO}$ to be almost spherical, and they considered this result as an indication that bonding in $\mathrm{MgO}$ can be treated as predominantly ionic.

\section{Comparison of Calculated and Observed Properties of $\mathrm{NiO}$}

The NiO specimens for the EELS experiments were prepared following the same procedure as in the case of $\mathrm{UO}_{2}$ described in the previous section. The near edge structure of the $\mathrm{O} \mathrm{K}$ electron energy loss spectrum of $\mathrm{NiO}$ is shown in Fig. 4. As in the case of $\mathrm{UO}_{2}$ the dipole selection rules pick out final states of p symmetry localized on oxygen sites.

Numerical calculations were performed using the LSDA + U method described above and using the Moruzzi-Janak-Williams exchange-correlation potential. The structure of the experimental EELS spectrum shown in Fig. 4 is dominated by the dipole ffilled $1 \mathrm{~s}\} \rightarrow\{$ empty $2 \mathrm{p}\}$ transitions (note that the presence of non-sphericity of the crystal field around oxygen sites in $\mathrm{NiO}$ does not alter this conclusion [45] since the expansion of the non-spherical part of the electron density starts from $l=4)$. In agreement with the results of previous LSDA calculations [7], we found that the ground state of nickel monoxide in LSDA is antiferromagnetic and insulating where the $0.5 \mathrm{eV}$ bandgap separates two bands of nickel d states. This LSDA result is known to be at odds with experimental data exhibiting a much larger bandgap and different ordering of bands [9, 18]. Fig. 4 shows that the O 2p DOS spectrum calculated using LSDA does not agree well with the experimental EELS spectrum. The peaks in the EELS spectrum are associated with hybridization between oxygen $2 \mathrm{p}$ and nickel $3 \mathrm{~d}$, $4 \mathrm{~s}$ and $4 \mathrm{p}$ states, respectively, and our analysis shows that the latter two peaks are practically unaffected by Hubbard correlations in the $3 \mathrm{~d}$ shell.

The separation between the two main peaks (i.e. the $\mathrm{O} 2 \mathrm{p}-\mathrm{Ni} 3 \mathrm{~d}$ and $\mathrm{O} 2 \mathrm{p}-\mathrm{Ni} 4 \mathrm{p}$ peaks) seen in the LSDA DOS curve is approximately $2 \mathrm{eV}$ larger than the separation between the same peaks in the experimental spectrum, and the spectral weight of the low-energy peak in the DOS calculated using LSDA is far too high. The equilibrium lattice constant $a_{\mathrm{LSDA}}=4.08 \AA$, although in better agreement with experiment than the LMTO-ASA value of $3.98 \AA$ [12], is still lower than the experimentally observed one of $4.17 \AA$.

Fig. 4 also shows the oxygen 2 p DOS calculated using the LSDA + U functional (6) for two values of $\bar{U}=6.2 \mathrm{eV}$ and $\bar{U}=8.0 \mathrm{eV}$, and for $\bar{J}=0.95 \mathrm{eV}$. The values of $\bar{U}=8.0 \mathrm{eV}$ and for $\bar{J}=0.95 \mathrm{eV}$ have been proposed by Anisimov and Gunnarsson [46]. Since the method used by Anisimov and Gunnarsson did not take into account the self- 

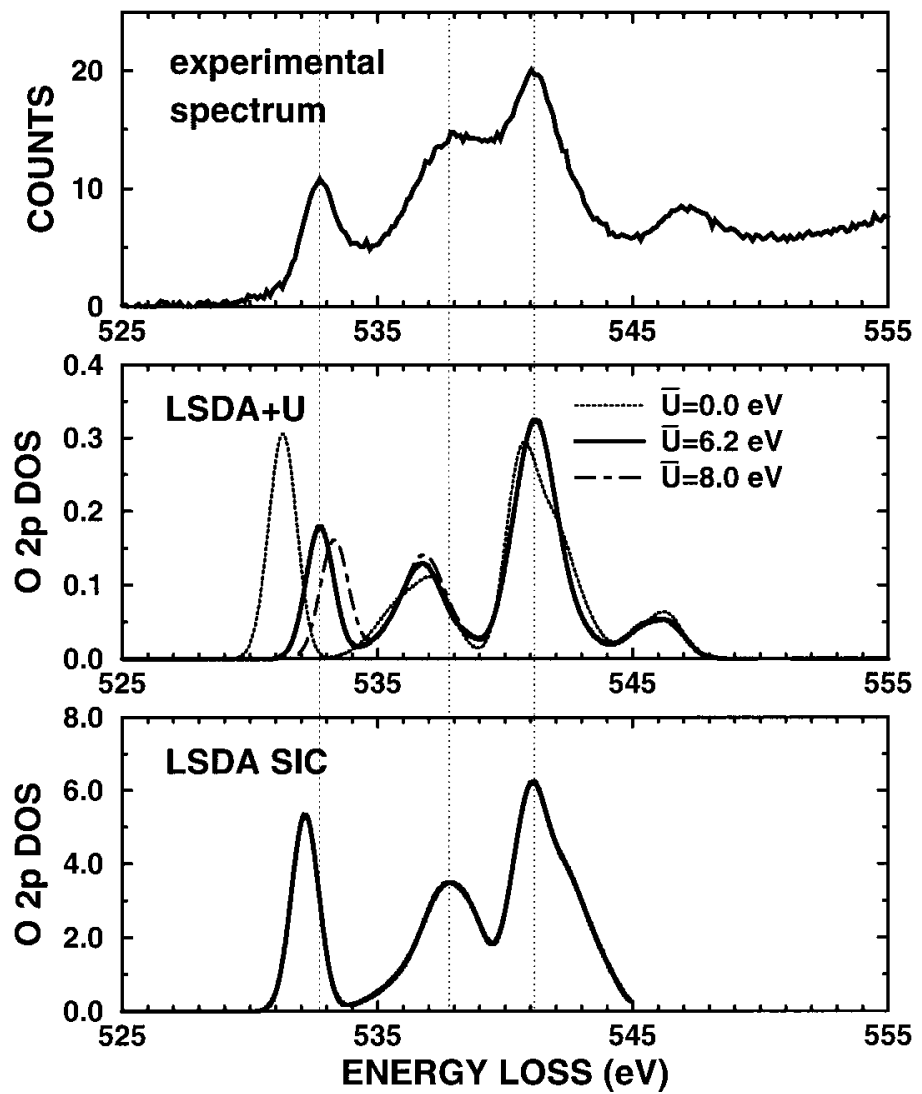

Fig. 4. Experimental electron energy loss oxygen $\mathrm{K}$ edge spectrum of $\mathrm{NiO}$ and the density of empty oxygen $2 \mathrm{p}$ states of $\mathrm{NiO}$ calculated by using LSDA $(\bar{U}=0)$, LSDA $+\mathrm{U}(\bar{U}=6.2$ and $8 \mathrm{eV})$ and self-interaction corrected LSDA

screening of d electrons, it is likely that the actual value of $\bar{U}$ is slightly smaller than $8 \mathrm{eV}$. By analysing how the separation between the two main peaks the spectrum of DOS shown in Fig. 6 depends on the value of $\bar{U}$, we concluded that the lower value of $\bar{U}=6.2 \mathrm{eV}$ agrees better with experimental data. For comparison, we mention the empirical value of $\bar{U}=6.7 \mathrm{eV}$ quoted in [18]. The equilibrium lattice constants found using the above values of $\bar{U}$ are $a_{\mathrm{LSDA}+\mathrm{U}}(\bar{U}=6.2 \mathrm{eV})=4.19 \AA$ and $a_{\mathrm{LSDA}+\mathrm{U}}(\bar{U}=8.0 \mathrm{eV})$ $=4.22 \AA$, and both of these values agree with experimental data better than the LSDA result.

Similarly to the LSDA + U, application of the SIC-LSDA to NiO leads to a substantial bandgap [26] of $3.15 \mathrm{eV}$, at the theoretical volume, which compares favourably both with the LSDA + U and observed gaps. Also the equilibrium lattice constant of $4.18 \AA$, calculated within the SIC-LSDA, is a substantial improvement on the LSDA value. In order to compare the EELS spectra and the LSDA + U results with the SIC-LSDA calculation, we calculated the SIC-LSDA density of one-particle oxygen $2 p$ states using the program described in detail in [26]. The spectrum of oxygen 2p DOS obtained from this calculation is shown in Fig. 4. By comparing the curves shown in Fig. 4 we find that the 


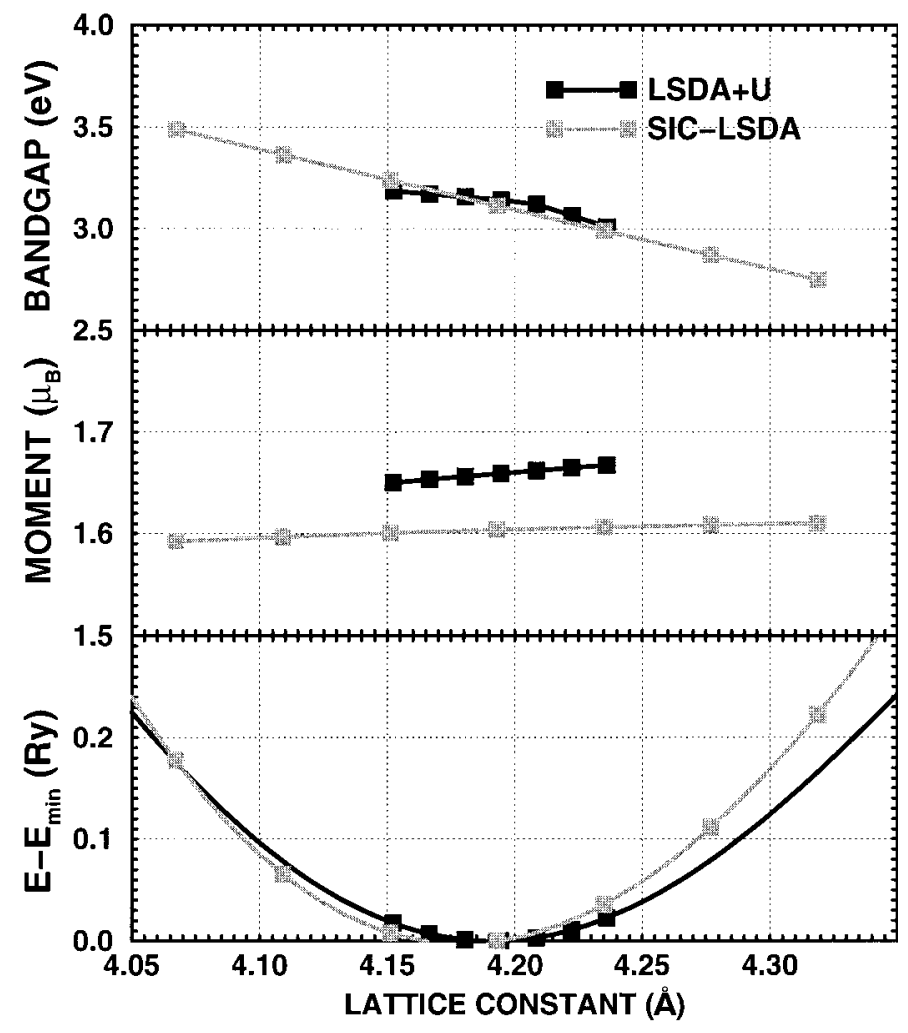

Fig. 5. The bandgap, the magnetic moment of a nickel ion in $\mathrm{NiO}$ and the total energy of the antiferromagnetic unit cell calculated using LSDA + U and SIC-LSDA and plotted as a function of the lattice constant. The observed size of the bandgap equals [9] $4.2 \mathrm{eV}$, the observed values of the magnetic moment of a nickel ion in $\mathrm{NiO}$ lie in the interval between [18] 1.66 and $1.90 \mu_{\mathrm{B}}$, and the equilibrium lattice constant equals $4.17 \AA$

introduction of the self-interaction correction in LSDA also slightly reduces the separation between the two main peaks in the spectrum of DOS and therefore improves the agreement between the calculated and experimentally observed EELS spectra compared with LSDA. We should say that although LSDA + U and SIC-LSDA methods are based on different physical assumptions, the fact that the calculated spectra of DOS exhibit similar trends suggests the need for orbital dependent potentials to describe compounds characterized by the presence of strong correlations between valence electrons.

To characterize the structural stability of the material, we have calculated the cohesive energy and elastic moduli of $\mathrm{NiO}$. Calculations were performed following the procedure described in [41] and involved the analysis of the dependence of the total energy on the positions of ions in the unit cell. Fig. 5 displays the result of two such calculations performed using the LSDA + U and SIC-LSDA approaches showing the total energy, the magnetic moment and the bandgap plotted as a function of the lattice constant. The numerical values calculated by LSDA + U and SIC-LSDA agree well both between themselves and with experimental data. It is interesting to note that according to both the LSDA + U and SIC-LSDA calculations the bandgap in NiO increases with increas- 


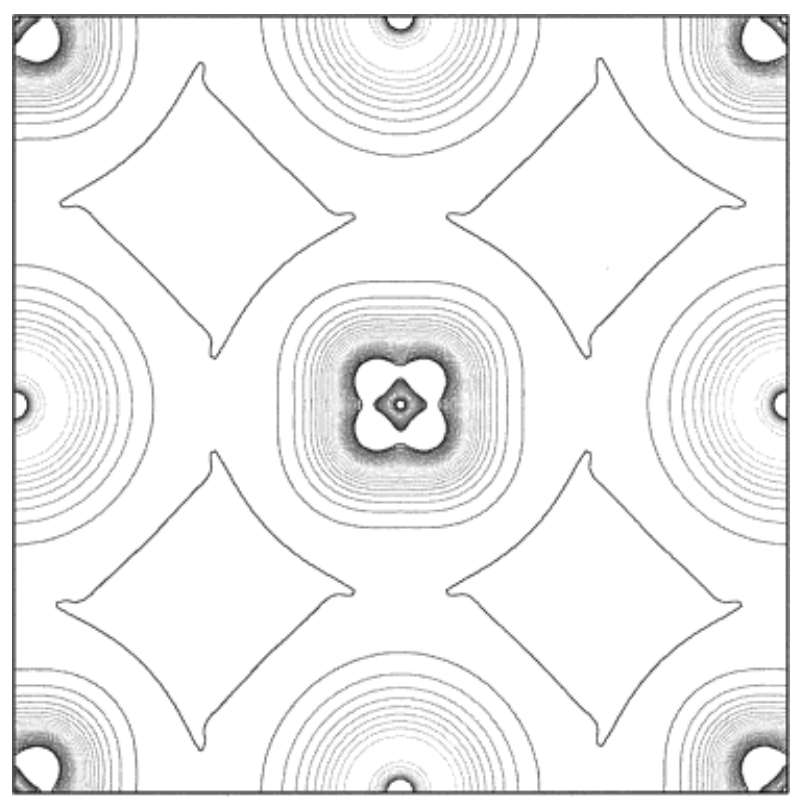

Fig. 6. The distribution of the total charge density in the (100) plane of $\mathrm{NiO}$ calculated by using the LSDA + U approach

ing pressure. The bandgap was also found to behave similarly in LSDA. We do not view this finding as contradicting the general principles stating that all compounds become metallic under sufficiently high external pressure. As the lattice constant $a_{0}$ decreases further the behaviour of the bandgap as a function of $a_{0}$ changes and it becomes an increasing function of $a_{0}$. The cohesive energy of $\mathrm{NiO}$ quoted in Table 1 was obtained by comparing the minimum of the LSDA $+\mathrm{U}$ curve shown in Fig. $5\left(E_{\min }=-6372.249 \mathrm{Ry}\right)$ with the energies of individual nickel and oxygen ions $E_{\mathrm{Ni}}=-3036.2203 \mathrm{Ry}$ and $E_{O}=-149.0551 \mathrm{Ry}$.

The calculated values of elastic moduli of $\mathrm{NiO}$ are listed in Table 1 together with the corresponding experimental data and with ab-initio Hartree-Fock values calculated by Towler et al. [47]. The data given in the table show that on balance the LSDA $+\mathrm{U}$ and SIC-LSDA values argree with experimental data better than the LSDA results.

To compare the distribution of charge in the unit cell of $\mathrm{NiO}$ with that of $\mathrm{UO}_{2}$ in Fig. 6 we plotted this distribution in the (100) plane calculated using the LSDA + U approach. In Fig. 6 four nickel ions are situated in the corners and in the centre of the image. Four oxygen ions occupy positions between nickel ions. In agreement with the results reported by Towler et al. [47] the distribution of charge around nickel sites shows a considerable degree of anisotropy. This illustrates a substantial role played by covalent effects in this oxide and illustrates the difference between the character of chemical bonding in $\mathrm{NiO}$ and $\mathrm{UO}_{2}$. Note that the distribution of charge around oxygen ions remains spherical in both cases.

\section{Conclusions}

We have reported results of an application of the LSDA + U method to the calculation of the one-particle densities of states and the total energy of crystalline uranium dioxide and nickel monoxide. We have found that by using a combined DFT-LSDA and UHF 
description of one-electron states it is possible to achieve reasonable agreement between the calculated and the experimentally measured electron energy loss spectra, and at the same time to evaluate parameters characterizing the structural stability of the material. We also compared the LSDA + U and experimental results with an SIC-LSDA calculation. We believe that the reported findings open the way towards carrying out more accurate ab-initio studies of surfaces and defects in transition metal and actinide oxides.

Acknowledgements We are grateful to Martin Castell, Alexander Liechtenstein, Dave Goddard, Andrew Briggs and Colin Humphreys for stimulating discussions. Computations were performed in the Materials Modelling Laboratory of the Department of Materials at the University of Oxford. Financial support of this work from BNFL is gratefully acknlowledged.

\section{References}

[1] V. F. Kiselev and O. V. Krylov, Adsorption and Catalysis on Transition Metals and Their Oxides, Springer-Verlag, Berlin 1989.

[2] G. J. Hutchins, C. S. Heneghan, I. D. Hudson, and S. H. Taylor, Nature 384, 341 (1996).

[3] N. F. Motт, Metal-Insulator Transitions, Taylor and Francis, London 1974.

[4] D. Baeriswyl, D. K. Campbell, J. M. P. Carmelo, F. Guinea, and E. Louis, (Eds.), The Hubbard Model, NATO ASI Ser. B, Vol. 343, Plenum Press, New York 1995

[5] A. Georges, G. Kotliar, W. Krauth, and M. J. Rozenberg, Rev. Mod. Phys. 68, 13 (1996).

[6] J. Yamashita and S. Asano, J. Phys. Soc. Jpn. 52, 3514 (1983).

[7] K. Terakura, A. R. Williams, T. Oguchi, and J. Kübler, Phys. Rev. Lett. 52, 1830 (1984); Phys. Rev. B 30, 4734 (1984).

[8] D. Adler, in: Solid State Physics, Ed. F. Seitz, D. Turnbull, and H. Ehrenreich, Vol. 21, Academic Press, New York 1968.

[9] G. A. Sawatzky and J. W. Allen, Phys. Rev. Lett. 53, 2339 (1984).

[10] T. Petit, B. Morel, C. Lemaignan, A. Pasturel, and B. Bigot, Phil. Mag. B73, 893 (1996).

[11] S. L. Dudarev, D. Nguyen Manh, and A. P. Sutton, Phil. Mag. B75, 613 (1997).

[12] O. K. Andersen, H. L. Skriver, H. Nohl, and B. Johansson, Pure Appl. Chem. 52, 93 (1980).

[13] O. Gunnarsson, D. D. Sarma, F. U. Hillebrecht, and K. Schönhammer, J. Appl. Chem. 63, 3676 (1988).

[14] S. Hüfner, Adv. Phys. 43, 183 (1994).

[15] M. R. Castell, P. L. Wincott, N. G. Condon, C. Muggelberg, G. Thornton, S. L. Dudarev, A. P. Sutton, and G. A. D. Briggs, Phys. Rev. B 55, 7859 (1997).

[16] S. L. Dudarev, A. I. Liechtenstein, M. R. Castell, G. A. D. Briggs, and A. P. SutTON, Phys. Rev. B 56, 4900 (1997).

[17] M. R. Castell, S. L. Dudarev, C. Muggelberg, A. P. Sutton, G. A. D. Briggs, and D. T. Goddard, J. Vac. Sci. Technol. A 16(3) (1998) in press.

[18] V. I. Anisimov, J. ZaAnen, and O. K. Andersen, Phys. Rev. B 44, 943 (1991).

[19] V. I. Anisimov, I. V. Solovyev, M. A. Korotin, M. T. Czyzyk, and G. A. Sawatzky, Phys. Rev. B 48, 16929 (1993).

[20] A. I. Liechtenstein, V. I. Anisimov and J. Zahnen, Phys. Rev. B 52, R5467 (1995).

[21] S. Y. Savrasov, Phys. Rev. B 54, 16470 (1996).

[22] E. Fradkin, Field Theories of Condensed Matter Systems, Chap. 2, Addison-Wesley Press, Reading, (Mass.) 1991.

[23] A. Kotani and T. Yamazaki, Prog. Theor. Phys. Suppl. 108, 117 (1992).

[24] J. P. Perdew and A. Zunger, Phys. Rev. B 23, 5048 (1981).

[25] R. O. Jones and O. Gunnarsson, Rev. Mod. Phys. 61, 689 (1989). 
[26] W. M. Temmerman, A. Svane, Z. Szotek, and H. Winter, Electronic Density Functional Theory: Recent Progress and New Directions, Eds. J. F. Dobson, G. Vignale, and M. P. DAS, Plenum, Press, New York 1997.

[27] A. Svane and O. Gunnarsson, Solid State Commun. 76, 851 (1990).

[28] A. Svane and O. Gunnarsson, Phys. Rev. Lett. 65, 1148 (1990).

[29] Z. Szotek, W. M. Temmerman, and H. Winter, Phys. Rev. B 47, 4029 (1993).

[30] M. Arai and T. Fujiwara, Phys. Rev. B 51, 1477 (1995).

[31] A. Svane, Phys. Rev. Lett. 68, 1900 (1992).

[32] W. M. Temmerman, Z. Szotek and H. Winter, Phys. Rev. B 47, 11533 (1993).

[33] W. R. Lambrecht and O. K. Andersen, Phys. Rev. B 34, 2439 (1996). O. K. Andersen, A. Paxton, M. SchilfgaArde, and O. Jepsen, unpublished.

[34] G. A. Botton, G. Y. Guo, W. M. Temmerman, and C. J. Humphreys, Phys. Rev. B 54, $1682(1996)$.

[35] X. Weng, P. Rez, and O. F. Sankey, Phys. Rev. B 40, 5694 (1989).

[36] F. M. F. de Groot, J. Faber, J. J. M. Michiels, M. T. Czyzyk, M. Abbate, and J. C. FugGle, Phys. Rev. B 48, 2074 (1993).

[37] J. C. Krupa and Z. GajeK, Europ. J. Solid State Inorg. Chem. 28, 143 (1991).

[38] J. S. GRIffith, The Theory of Transition-Metal Ions, Cambridge University Press, 1961 (pp. 73 to 79 ).

[39] Y. Baer and J. Schoenes, Solid State Commun. 33, 885 (1980).

[40] P. J. Kelly and M. S. S. Brooks, J. Chem. Soc. Faraday Trans. II 83, 1189 (1987).

[41] D. M. Sherman, J. Geophys. Res. 98, 19795 (1993).

[42] Landolt-Börstein Numerical Data and Functional Relationships in Science and Technology, Group III: Crystal and Solid State Physics, Vol. 11, Ed. K.-H. Hellwege, Springer-Verlag, Berlin 1979.

[43] J. Faber, JR. and G. H. Lander, Phys. Rev. B 14, 1151 (1976).

[44] J. M. Zuo, M. O'Keeffe, P. Rez, and J. C. H. Spence, Phys. Rev. Lett. 78, 4777 (1997).

[45] S. Sugano, Y. Tanabe, and H. Kamimura, Multiplets of Transition-Metal Ions in Crystals, Academic Press, New York 1970.

[46] V. I. Anisimov and O. Gunnarsson, Phys. Rev. B 43, 7570 (1991).

[47] M. D. Towler, N. L. Allan, N. M. Harrison, V. R. Saunders, W. C. Mackrodt, and E. Apra, Phys. Rev. B 50, 5041 (1994).

[48] T. Sasaki, Phys. Rev. B 54, R9581 (1996). 
